\title{
Epigenetic Effects of Nutrients and the Promise for Refined Cardiovascu- lar Disease Prevention
}

\author{
Gertrud Lund $^{1}$ and Silvio Zaina, ${ }^{2, *}$ \\ ${ }^{I}$ Cinvestav Campus Guanajuato, Irapuato, Mexico; ${ }^{2}$ Department of Medical Research, Division of Health Sciences, \\ Campus Leon, University of Guanajuato, Leon, Mexico
}

\begin{abstract}
It is clear that dietary factors can induce epigenetic changes - i.e. can alter patterns of DNA methylation and histone posttranslational modifications in the genome. At least part of the epigenetic effects elicited by diet in infants may result in long-lasting changes in gene expression within an individual's lifetime and, at least in animal models, transgenerationally. Therefore, these epigenetic modifications can be regarded as early molecular events of potentially critical relevance to vascular disease prevention. A comprehensive description of dietary factor-induced changes in the epigenome - including both protective and risk-generating factors - is needed to appreciate the extent and relevance of these early molecular events. A second critical goal yet to be achieved is the description of the epigenome of cell types participating in atherogenesis. By combining these two approaches, research promises to generate information that can be translated into improved vascular disease prevention. Here, we review recent advances in the field of epigenetics, dietary factors and vascular disease.
\end{abstract}

Keywords: Epigenetics, nutrition, gene-nutrient interaction.

"DNA is not destiny"

R.A. Waterland and K.B. Michels, ref. [1]

"You may have obese parents, but if you live a healthy life you will make new genes so that your sons will be healthy" Stated in a popular medicine radio program

\section{EPIGENETICS}

Epigenetics studies mechanisms of gene regulation that are dependent on chromatin structure. Nuclear chromatin is composed of DNA wrapped around a hetero-octamer of histones called "core histones", which include two copies of each histone type 2A, 2B, 3 and 4 . One core histone octamer and the DNA wrapped around it constitute a nucleosome, the fundamental repetitive unit of the chromatin polymer. Chromatin can be organised as a compact structure that generally includes inactive genes (non-permissive chromatin), or a more relaxed structure (permissive chromatin) - (Fig. (1)). Genes embedded in permissive chromatin are accessible to the transcription machinery and are therefore potentially active [2].

The two types of chromatin are associated with specific epigenetic modifications of the chromatin itself. The best understood epigenetic modifications are DNA methylation and histone posttranslational modifications. DNA methylation in mammals occurs almost exclusively at position 5 of cytosine residues in a $\mathrm{CpG}$ dinucleotide context to yield 5methyldeoxycytidine $(5 \mathrm{mdC})$. The various known histone modifications include acetylation, methylation, phosphorylation and sumoylation. A specific combination of epigenetic

*Address correspondence to this author at the Department of Medical Research, Division of Health Sciences, Campus Leon, University of Guanajuato, 20 de Enero no.929, 37000 Leon, Gto., Mexico; Tel./Fax: +52 477 714 3812; E-mail: szaina@leon.ugto.mx modifications determines or reflects the transcriptional status of a given DNA regions. For example, DNA hypomethylation, histone hyperacetylation and hypomethylation of lysine 20 of histone 4 (H4K20) are modifications generally associated with active DNA regions, whereas the reciprocal modifications are present in inactive regions of the genome [2] (Fig. (1)).

The combination of specific epigenetic modifications present in any given cell nucleus is collectively referred to as epigenome. The epigenome is at least in part a plastic entity, in the sense that patterns of DNA methylation and histone modifications are modified during growth and differentiation. One extreme example of this phenomenon is the widespread erasure and re-establishment of DNA methylation patterns during mammalian embryo development, clearly illustrating the reversible character of epigenetic changes [3]. Epigenetic patterns differ between different cell types, implying that different epigenomes coexist in a genetically uniform organism - i.e. with an identical genome in all cells, with the exception of antibody-producing cells [4]. Indeed, the degree of plasticity is a major difference between the epigenome - a fluctuating entity during development and cell specialization - and the genome - changing through mutations, which are generally irreversible events. Yet, epigenetic patterns are generally inherited during mitosis and meiosis, indicating that the epigenome shows a high degree of stability at least in a homogeneous cell population. 


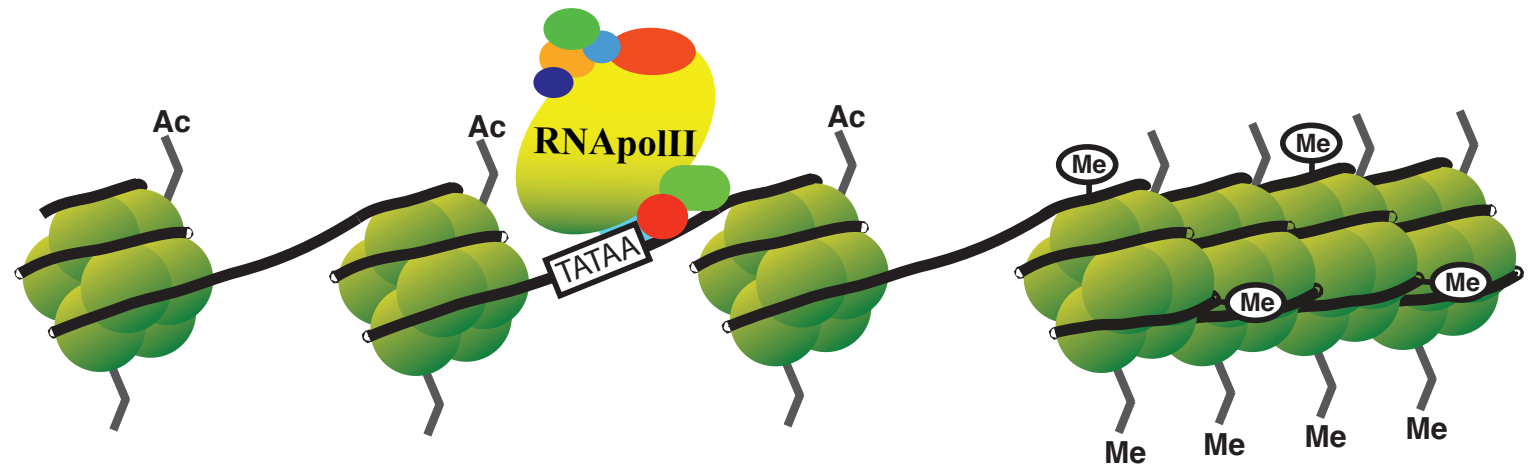

Fig. (1). Schematic representation of chromatin structure. DNA (black line) is wrapped around octamers composed of two copies of each histone H2A, H2B, H3 and H4 (green spheres). A histone octamer and the DNA associated with constitute a nucleosome, or the fundamental unit of the chromatin polymer. Nucleosomes can be arranged in a relatively open architecture accessible to transcription factors (permissive chromatin, left) or in a compact one (non-permissive chromatin, right). A schematic RNA polymerase II - transcription factor complex bound to a promoter is shown in permissive chromatin. Chromatin architectures are specifically associated with histone posttranslational modifications and DNA methylation status. Specific histone residues are hyperacetylated (Ac) and hypermethylated (Me) in permissive and non-permissive chromatin, respectively. DNA is generally hypermethylated in non-permissive chromatin (Me).

\section{VASCULAR DISEASE: A CENTRAL ROLE FOR EPI- GENETIC GENE REGULATION?}

The notion that alterations in the epigenome can play a significant role in numerous diseases such as cancer, mental disorders and atherosclerosis has rapidly gained ground in recent years $[5,6]$. In the case of cancer, a frequently observed epigenetic change is global loss of 5mdC (DNA hypomethylation) and concomitant hypermethylation of a subset of CpG-rich regions called $\mathrm{CpG}$ islands, typically located in promoter regions.

It is widely believed that changes in the population's exposure to diet- and life style-related factors underlie the recent epidemics of metabolic diseases such as obesity, diabetes, metabolic syndrome and vascular complications, and that genetic components play an accessory, if not limited, role in these phenomena. Specifically, exposure to specific dietary factors during early life can determine adult cardiovascular disease (CVD) risk. Noticeable examples are the protective role of breast-feeding extending through adult life and the transgenerational effect of diet on CVD risk [7, 8]. Both observations are difficult to interpret without taking into account epigenetic mechanisms of gene regulation - in this case the imposition of stable chromatin states, whether by direct chromatin remodelling or by the induction of biochemical changes that produce long-lasting chromatin states. One example of the latter may be an abnormal production of a biochemical factor - a hormone - resulting in constitutive transcription and permissive chromatin at target genes (discussed in ref. [9]) - (Fig. (2)). Thus, a permanent, long-term acting epigenetic "mark" may be imposed on specific genes by transient exposure to dietary or environmental factors during early life or by continuous exposure in adult life [10]. A demonstrated epigenetic basis for CVD, together with the potentially reversible nature of epigenetic modifications, would open new prospects for correcting aberrant gene expression with drugs that target the epigenome. This line of reasoning has been shown to be valid in the case of cancer and has produced interesting candidate epigenome-targeting anti-tumour drugs [11]. By contrast, research on the epigenetics of CVD is still in its infancy.

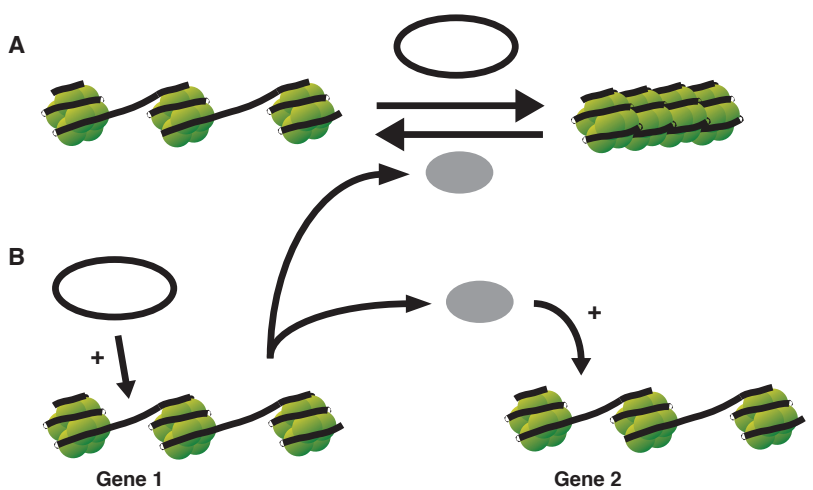

C

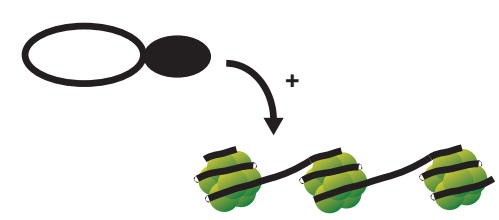

Fig. (2). Central role for chromatin modifications and epigenetics in gene regulation by nutritional/environmental factors. One such factor is represented by an open ellipse. Three cases are schematically shown. A, the factor is a chromatin modifier that binds to chromatin and causes its transition between permissive and nonpermissive architectures. B, the factor regulates transcription of gene 1. The product of gene 1 (grey ellipse) can be a chromatin modifier or a factor regulating gene 2 in trans or in a different cell, for example a hormone. $\mathrm{C}$, the factor primarily induces a biochemical change (for example induces a complex with a second factor, black ellipse) to activate a target gene. In B and C, transcription can induce a "memory" determining a stable permissive chromatin state. Any transient exposure to a specific factor has to induce a stable chromatin architecture to achieve a long-lasting gene expression pattern. Even if a factor's effects are short lasting, they depend on specific pre-existing chromatin architecture at target genes.

\section{EPIGENETIC ABNORMALITIES DURING THE NAT- URAL HISTORY OF ATHEROSCLEROSIS}

The idea that epigenetic mechanisms of gene regulation underlie CVD has been put forward for the first time by P.E. 
Newman. The author based his hypothesis on available studies claiming that folate, vitamin B6 and B12 deficiency causes atherosclerosis. By linking the latter data with biochemical evidence that folate and vitamin B are necessary to sustain synthesis of S-adenosyl methionine (SAM), a universal donor of methyl groups in DNA methylation reactions, Newman proposed that vitamin deficiency leads to CVD through DNA hypomethylation [12]. Subsequent work confirmed some, but not all of Newman's predictions. Studies addressing the role of folate in CVD yielded controversial results, indicating that the causal relationship between vitamin deficiency, atherogenesis and aberrant DNA methylation is not as straightforward as previously thought (reviewed in ref. [13-15]). On the other hand, as anticipated in Newman's seminal paper [12], a number of studies detected abnormal DNA methylation patterns in atherosclerotic lesions in humans and animal models including rabbits and atherosclerosis-prone, hyperlipidaemic apolipoprotein E (ApoE)deficient mice. We reported that epigenetic abnormalities precede the formation of histologically detectable atherosclerotic lesions, as pre-atherosclerotic aortas of young ApoEdeficient mice show a mix of DNA hyper- and hypomethylation [6]. Furthermore, a few independent studies demonstrated a global loss of DNA methylation in advanced atherosclerotic lesions $[6,16,17]$. It is unclear whether DNA hypomethylation in atherosclerosis is the consequence of passive loss of methyl groups due to inefficient maintenance of DNA methylation patterns in intensely proliferating vascular lesion cells, the result of an active de- methylation process, or a combination of both. To date, the only proposed mechanisms for DNA hypomethylation in CVD centres around homocysteine (Hcy). The interest in Hcy stems from at least two observations. First, at a biochemical level, excess Hcy causes the accumulation of its own precursor Sadenosyl-homocysteine (SAH), an inhibitor of DNA methyltransferase enzymes [18]. Second, hyperhomocysteinaemia has been implicated in atherogenesis, although the results of clinical studies have not been entirely consistent [19]. Therefore, it has been hypothesized that the main consequence of hyperhomocysteinaemia is DNA hypomethylation via a reduction of SAM bioavailability. This attractive model is supported by the association between hyperhomocysteinaemia, reduced SAM levels and peripheral blood cell DNA hypomethylation that was reported by independent studies in humans $[20,21]$. These findings are backed by observations in experimental animal models, showing that mice deficient in methylenetetrahydrofolate reductase, a crucial enzyme in the biochemical pathway that generates SAM, show DNA hypomethylation and vascular lipid deposits macroscopically similar to early atherosclerotic lesions [22]. At molecular level, a role for Hcy in chromatin regulation has been recently addressed by Jiang and colleagues [23]. The study showed that Hcy causes transcriptional repression of the extracellular superoxide dismutase gene and the appearance of non-permissive chromatin markers in the promoter of that gene in monocytes. Similar results were obtained for the fibroblast growth factor 2 genes in endothelial cells [24]. Despite all supporting evidence, the Hcy hypothesis shows weaknesses and pending issues to be addressed. First, Hcy may primarily induce cell proliferation and therefore induce DNA hypomethylation simply as a secondary consequence of defective maintenance of DNA methylation in highly pro- liferating advanced atherosclerotic lesion cells as proposed for cancer [25]. Since proliferating SMC represent a significant portion of elevated fibrocellular lesions, it is not surprising that the measurement of total lesion DNA methylation levels yields hypomethylation. Second, if Hcy plays a pivotal role in atherosclerotic lesion DNA hypomethylation, then hyperhomocysteinaemia is a prerequisite for that epigenetic anomaly. This prediction is contradicted by the observation that ApoE-deficient mutant mice, a widely used model of hyperlipidaemia-induced atherosclerosis, display concomitant lesion DNA hypomethylation and hypohomocysteinaemia - i. e. Hcy levels 30-50\% lower than human counterpart [26]. The issue would be clarified by addressing the question whether DNA hypomethylation is restricted to the subset of hyperhomocysteinaemic atherosclerotic patients. Taken together, the available data suggest that hyperhomocysteinaemia per se is not an underlying cause of DNA hypomethylation in atherosclerosis, although it is possible that Hcy operates as an active modifier of DNA methylation in synergy with other yet unidentified factors. This goes without saying that, as we mentioned above, the relevance of hyperhomocysteinemia in atherosclerosis has been questioned [19].

The majority of studies published so far compared whole lesions or affected arterial segments with normal tissue, thus lacking detail at the cell-type level. Clearly, an analysis at smooth muscle cell (SMC) and macrophage level, two highly represented cell types in lesions, is greatly needed. One step in this direction has been taken by studies analysing the methylation status of estrogen receptor genes $(E S R)$. The importance of ESR in this context stems from the atheroprotective properties of estrogens and from the observation that methylation at the ESR genes increases with ageing, an independent risk factor for atherosclerosis [27]. Two of such studies detected hypermethylation of the estrogen receptor 2 (beta) gene (ESR2) in both cultured vascular SMC and endothelial cells, whereas estrogen receptor alpha gene (ESRI) hypermethylation was apparently restricted to the SMC [28, 29]. The data clearly draw a molecular parallel between ageing and atherosclerosis, reinforcing the idea of accelerated local ageing occurring at vascular lesion site.

In summary, associative studies consistently indicated that epigenetic aberrations occur during the natural history of atherosclerosis. Future tasks that need to be completed to demonstrate an epigenetic basis for atherosclerosis include solid retrospective studies matching historical epigenetic profiles with disease outcome, backed by the compilation of a lesion's epigenome at cell type level.

\section{EPIGENETICS IN INFLAMMATION}

Given the pivotal role of inflammation in atherosclerosis, the question of whether pro-inflammatory signals cause changes in DNA methylation may provide important insights into the relevance of epigenetics in the disease. Recent work by Stenvinkel and colleagues addressed the association between inflammation and peripheral blood leukocyte global DNA methylation status in chronic kidney disease patients [30]. The study showed a robust association between inflammatory markers and global DNA hypermethylation. Importantly, the authors suggest that peripheral blood DNA hypermethylation could become an inflammatory disease 
risk predictor or diagnostic, particularly as it is detectable in such an accessible sample. These findings are consistent with the observation that the potent pro-inflammatory factor interleukin 6 promotes the translocation of DNMT1, a DNA methyltransferase, from cytoplasm to nucleus [31]. Further work is needed to establish how common the association between DNA hypermethylation and inflammation is in different diseases. At any rate, the occurrence of global DNA hypomethylation in advanced atherosclerosis indicates that such an association is confined to either specific diseases or specific disease stages.

\section{NUTRITIONAL FACTORS AND THE EPIGENOME}

If the studies discussed above clearly demonstrate that epigenetic abnormalities are associated with the natural history of atherosclerosis and with inflammation, the question that needs to be addressed next is what the cause of those epigenetic changes is. In particular, could the epigenome be modified by nutritional and environmental factors? Is there any clear model systems describing such phenomena and what is their relevance for CVD? In this and the following paragraph we provide some answers to these questions by reviewing some of the most relevant studies.

The first clear example of a simple dietary factor affecting DNA methylation and gene expression has been the mouse carrying the $A^{v y}$ agouti allele [32]. $A^{v y}$ females produce offspring with coat colour ranging from yellow to brown (pseudoagouti). The authors first described the molecular events underlying this phenotypic variability. Coat colour is associated with the methylation status of a transposon inserted 5' to the agouti gene in the $A^{v y}$ allele. In yellow mice, the transposon is hypomethylated and acts as a potent promoter to cause ectopic expression of the agouti gene. Since the agouti gene product is involved in metabolic regulation in the hypothalamus, yellow mice display metabolic abnormalities including obesity and diabetes. The transposon of $A^{v y}$ is more methylated in intermediate coat colour mice and completely methylated in pseudoagouti offspring. The pattern of agouti expression is more and more normal as the transposon DNA methylation increases. Subsequently, the authors sought to correct the ectopic expression of the $A^{v y}$ allele by supplementing the mother's diet with a factor capable of increasing DNA methylation activity. Folate was the obvious choice, and indeed $A^{v y}$ females feeding a folate-fortified diet generated offspring with coat colour distribution strongly shifted towards pseudoagouti. This elegant work shows not only that diet can significantly affect gene expression and the epigenome, but also that these effects take place in utero and are therefore transgenerational. Similar results were obtained by the same group in the Axin mouse model [33].

A recent study conducted in honeybee (A. mellifera) offers another dramatic example of dietary control of the epigenome [34]. Genetically identical female larvae can develop into fertile queens or sterile workers depending on whether fed with royal jelly, implying that epigenetic changes controlled by a dietary factor underlie this phenotype switch. Indeed, females in which the expression of the DNA methyltransferase DNMT3 was silenced developed mostly as fertile queens, irrespective of dietary regime. Cru- cially, the DNA methylation status of the dynactin p62 gene - an important player in honeybee larval development - was remarkably similar in in-hive reared queen larvae and in DNMT3-deficient in vitro reared ones. These observations demonstrate that the predominant effect of royal jelly is the imposition of a specific de novo DNA methylation pattern through the DNMT3 enzyme. Importantly, this is an example of a dietary factor acting at a specific time window during an organism's early development yet producing stable, longterm physiological effects that extend into adult life.

Obviously, the studies described in this paragraph provide important conceptual frames to understand long-lasting effects of childhood nutrition - for example breast milk - and even transgenerational effects of diet on metabolic disease risk reported in humans [35]. These are certainly fascinating hypotheses, but is there any example of epigenetic changes induced by factors of dietary origin involved in atherogenesis? To date, the literature reports two examples. First, our work demonstrated that a mix of human native very low-, low- and high density lipoproteins (VLDL, LDL and HDL, respectively) induce de novo DNA methylation in cultured human THP-1 macrophages, and a comparatively HDL-poor lipoprotein mix induced a stronger response than a HDL-rich one [6]. In addition, lipoproteins induced two modifications of histone 4 (H4) that generally accompany DNA hypermethylation in non-permissive chromatin, i.e. hypoacetylation and lysine 20 (H4K20) hypermethylation [6]. Second, arachidonic acid induced DNA hypermethylation in endothelial cells, suggesting that the organism's fatty acid pool may regulate vascular tissue homeostasis by imposing epigenetic changes [36].

\section{NUTRITION, THE ENVIRONMENT, EPIGENETICS AND VASCULAR DISEASE: CHALLENGES FOR CVD PREVENTION IN TRANSITION COUNTRIES}

One of the most important changes in global health at the end of the $20^{\text {th }}$ century is the emergence of so-called "transition" or "westernised" countries characterised by concomitant marked economic growth, shift from traditional to western lifestyle and dramatically increased incidence of metabolic diseases and CVD. One spectacular negative outcome of this phenomenon is that obese individuals outnumber malnutrition sufferers worldwide [37]. Transitional countries offer interesting opportunities to study and interpret the numerous effects of nutritional and environmental lifestyle on the epigenome. First, they provide yet another proof that genetics alone cannot help understanding the current metabolic disease epidemics. The adverse effects of nutrition and environment are very likely to be caused by changes in the epigenome, while genetic polymorphisms are additional if not subordinate factors that can modulate metabolic disease predisposition. Second, transitional countries often present a cluster of factors with proven or strongly suspected activity as epigenome modifiers. Commonly, one such cluster is the availability of extremely cheap calories contained in nontraditional food, combined with severe pollution often accompanying economic development in the absence of rigorous legislative regulation. Mexico is in many ways a representative example. The country has not been spared from a new conquista represented by the invasion of ready-made food and refreshments marketed by strong international 
companies that replaced or at best significantly compete with traditional dishes. One potential effect of these changes is the exposure to unprecedented dietary factors - trans fatty acids, interesterified lipids - with equally unprecedented effects on gene expression, let alone the synergy with a more sedentary lifestyle. If the epigenome is as sensitive to dietary factors as it is believed, a stable change of diet must result in changes in gene expression, presumably causing a combination of neutral, adverse, favourable and compensatory effects. The emerging evidence presented above describing lipids as potential epigenome modifiers, suggest that appropriate studies on the epigenetic effects of westernised food components should be undertaken. In addition, the Mexican food market is shaped by the imperative to defeat malnutrition, a problem that is now confined to only specific areas of the country or converted itself into a combination of over- and malnutrition. One prominent initiative is the addition of folic acid and other vitamins into nearly all food items available on supermarket shelves. As outlined above, folic acid produces marked epigenetic changes in animal models, and corresponding effects in humans are likely [38]. Two studies conducted in the US investigated the effects on folic acidfortified food consumption, DNA methylation and cervical cancer. The results indicate that fortified food consumption does not significantly alter global DNA methylation patterns, although it increases DNA methyltransferase expression [39, 40]. These data do not rule out the possibility that continued exposure to folic acid causes subtle changes in DNA methylation that are not detectable by global epigenome analysis but may still be biologically significant. Again, populationbased studies on the effects of prolonged exposure to fortified food are warranted. Another potential source of epigenetic modifications is poor quality of the environment. The interest on epigenetic effects of pollutants is strong but still nascent, with only few examples reported in the literature. Arsenic can be considered an illustrative example, as epigenetic mechanisms in arsenic-induced pathologies including CVD are suspected or have been investigated, and arsenic contamination of surface water is a common problem in Mexico [41-44].

Table 1. Overview of Selected Epigenome Modifiers

\begin{tabular}{|c|l|c|}
\hline \multicolumn{1}{|c|}{ Factor } & \multicolumn{1}{|c|}{$\begin{array}{c}\text { Effect on DNA } \\
\text { Methylation }\end{array}$} & Ref. \\
\hline Lipoproteins & hypermethylation & {$[6]$} \\
\hline Vitamins & \multicolumn{2}{|c|}{} \\
\hline vit. D & hypermethylation & {$[46]$} \\
\hline pro vitamin A & hypermethylation & {$[36]$} \\
\hline folic acid & hypermethylation & {$[47]$} \\
\hline Antibiotics & hypermethylation & {$[45]$} \\
\hline Arsenic & hypermethylation & {$[48]$} \\
\hline
\end{tabular}

The selected examples discussed above indicate that the human population is exposed to a cocktail of potential and proven epigenome modifiers, which may significantly affect human health and vascular disease risk in particular (Table 1). Arguably, the issue is a burning one especially in transition countries but does not spare any population group. It follows that understanding the interaction between genes and nutritional/environmental factors is a priority in basic and medical research and is likely to contribute to vascular disease prevention and therapy. In particular, current research indicates that nutrient- and environment-gene interaction in utero and in childhood is a major determinant of adult chronic diseases, and suggests that health policy makers should translate research in that area into appropriate vascular disease prevention policies. Epigenetics may provide unique experimental and conceptual instruments to assist in these efforts.

\section{CONCLUSIONS}

The two citations presented at the beginning of this review show that the idea of genes being shaped by the environment and diet during an individual's lifetime and transgenerationally is not only taken seriously by modern science but it also roots in elementary knowledge about interactions between one's health and the environment. Epigenetics may help to interpret the multifactorial origin of vascular diseases. The challenge is now for epigenetists and physicians to convey that idea in an objective and effective way.

\section{REFERENCES}

[1] Waterland RA, Michels KB. Epigenetic epidemiology of the developmental origins hypothesis. Annu Rev Nutr 2007; 27: 363-88.

[2] Berger SL. The complex language of chromatin regulation during transcription. Nature 2007; 447: 407-12.

[3] Feinberg AP. Phenotypic plasticity and the epigenetics of human disease. Nature 2007; 447: 433-40.

[4] Lund G, Andersson L, Lauria M, et al. DNA methylation polymorphisms precede any histological sign of atherosclerosis in mice lacking apolipoprotein E. J Biol Chem 2004; 279: 29147-54.

[5] Turck D. Later effects of breastfeeding practice: the evidence. Nestle Nutr Workshop Ser Pediatr Program 2007; 60: 31-9.

[6] Pembrey ME, Bygren LO, Kaati G, et al. Sex-specific, male-line transgenerational responses in humans. Eur J Hum Genet 2006; 14 : 159-66.

7] Zaina S, Lund G. Epigenetics and Cardiovascular Disease. In: Esteller, M. Ed. Epigenetics in Biology and Medicine. Taylor \& Francis Group LLC 2008; in press.

Gallou-Kabani C, Junien C. Nutritional epigenomics of metabolic syndrome: new perspective against the epidemic. Diabetes 2005; 54: 1899-906.

Newman PE. Can reduced folic acid and vitamin B12 cause deficient DNA methylation producing mutations which initiate atherosclerosis? Med Hypotheses 1999; 53: 421-4.

Carlsson CM. Homocysteine lowering with folic acid and vitamin B supplements: effects on cardiovascular disease in older adults. Drugs Aging 2006; 23: 491-502.

Mangoni AA. Folic acid, inflammation, and atherosclerosis: false hopes or the need for better trials? Clin Chim Acta 2006; 367: 119 .

Davis N, Katz S, Wylie-Rosett J. The effect of diet on endothelial function. Cardiol Rev 2007; 15: 62-6.

Laukkanen MO, Mannermaa S, Hiltunen MO, et al. Local hypomethylation in atherosclerosis found in rabbit ec-sod gene. Arterioscler Thromb Vasc Biol 1999; 19: 2171-8.

Hiltunen MO, Turunen MP, Häkkinen TP, et al. DNA hypomethylation and methyltransferase expression in atherosclerotic lesions. Vasc Med 2002; 7: 5-11.

[15] Castro R, Rivera I, Blom HJ, et al. Homocysteine metabolism, hyperhomocysteinaemia and vascular disease: an overview. J Inherit Metab Dis 2006; 29: 3-20. 
[16] Yi P, Melnyk S, Pogribna M, et al. Increase in plasma homocysteine associated with parallel increases in plasma Sadenosylhomocysteine and lymphocyte DNA hypomethylation. J Biol Chem 2000; 275: 29318-23.

[17] Ingrosso D, Cimmino A, Perna AF, et al. Folate treatment and unbalanced methylation and changes of allelic expression induced by hyperhomocysteinaemia in patients with uraemia. Lancet 2003; 361: 1693-9.

[18] Chen Z, Karaplis AC, Ackerman SL, et al. Mice deficient in methylenetetrahydrofolate reductase exhibit hyperhomocysteinemia and decreased methylation capacity, with neuropathology and aortic lipid deposition. Hum Mol Genet 2001; 10: 433-43.

[19] Jiang Y, Jiang J, Xiong J, et al. Homocysteine-induced extracellular superoxide dismutase and its epigenetic mechanisms in monocytes. J Exp Biol 2008; 211: 911-20.

[20] Chang PY, Lu SC, Lee CM, et al. Homocysteine inhibits arterial endothelial cell growth through transcriptional down regulation of fibroblast growth factor-2 involving G protein and DNA methylation. Circ Res 2008; 102: 933-41.

[21] Kimura F, Seifert HH, Florl AR, et al. Decrease of DNA methyltransferase 1 expression relative to cell proliferation in transitional cell carcinoma. Int J Cancer 2003; 104: 568-78.

[22] Troen AM, Lutgens E, Smith DE, et al. The atherogenic effect of excess methionine intake. Proc Natl Acad Sci USA 2003; 100: 15089-94.

[23] Issa JP. Aging, DNA methylation and cancer. Crit Rev Oncol Hematol 1999; 32: 31-43.

[24] Post WS, Goldschmidt-Clermont PJ, Wilhide CC, et al. Methylation of the estrogen receptor gene is associated with aging and atherosclerosis in the cardiovascular system. Cardiovasc Res 1999; 43: 985-91

[25] Kim J, Kim JY, Song KS, et al. Epigenetic changes in estrogen receptor beta gene in atherosclerotic cardiovascular tissues and invitro vascular senescence. Biochim Biophys Acta 2007; 1772: 72 80 .

[26] Stenvinkel P, Karimi M, Johansson S, et al. Impact of inflammation on epigenetic DNA methylation - a novel risk factor for cardiovascular disease? J Intern Med 2007; 261: 488-99.

[27] Hodge DR, Cho E, Copeland TD, et al. IL-6 enhances the nuclear translocation of DNA cytosine-5-methyltransferase 1 (DNMT1) via phosphorylation of the nuclear localization sequence by the AKT kinase. Cancer Genomics Proteomics 2007; 4: 387-98.

[28] Waterland RA, Jirtle RL. Transposable elements: targets for early nutritional effects on epigenetic gene regulation. Mol Cell Biol 2003; 23: 5293-300.

[29] Waterland RA, Dolinoy DC, Lin JR, et al. Maternal methyl supplements increase offspring DNA methylation at Axin Fused. Genesis 2006; 44: 401-6.

[30] Kucharski R, Maleszka J, Foret S, et al. Nutritional control of reproductive status in honeybees via DNA methylation. Science 2008; 319: 1827-30.

[31] Pembrey ME. Time to take epigenetic inheritance seriously. Eur J Hum Genet 2002; 10: 669-71.
[32] Kiec-Wilk B, Polus A, Mikolajczyk M, et al. Beta-carotene and arachidonic acid induced DNA methylation and the regulation of pro-chemotactic activity of endothelial cells and its progenitors. $\mathbf{J}$ Physiol Pharmacol 2007; 58: 757-66.

[33] $10^{\text {th }}$ International Congress on Obesity, Sidney, Australia, 2006.

[34] Choi SW, Friso S, Keyes MK, et al. Folate supplementation increases genomic DNA methylation in the liver of elder rats. $\mathrm{Br}$ Nutr 2005; 93: 31-5

[35] Piyathilake CJ, Azrad M, Jhala D, et al. Mandatory fortification with folic acid in the United States is not associated with changes in the degree or the pattern of global DNA methylation in cells involved in cervical carcinogenesis. Cancer Biomark 2006; 2: 25966.

[36] Piyathilake CJ, Celedonio JE, Macaluso M, et al. Mandatory fortification with folic acid in the United States is associated with increased expression of DNA methyltransferase-1 in the cervix. Nutrition 2008; 24: 94-9.

[37] Vahter ME. Interactions between arsenic-induced toxicity and nutrition in early life. J Nutr 2007; 137: 2798-804.

[38] Salnikow K, Zhitkovich A. Genetic and epigenetic mechanisms in metal carcinogenesis and cocarcinogenesis: nickel, arsenic, and chromium. Chem Res Toxicol 2008; 21: 28-44

[39] Tchounwou PB, Wilson B, Ishaque A. Important considerations in the development of public health advisories for arsenic and arsenic-containing compounds in drinking water. Rev Environ Health 1999; 14: 211-29.

[40] Ongley LK, Sherman L, Armienta A, et al. Arsenic in the soils of Zimapán, Mexico. Environ Pollut 2007; 145: 793-9.

[41] Schmitt F, Oakeley EJ, Jost JP. Antibiotics induce genome-wide hypermethylation in cultured Nicotiana tabacum plants. J Biol Chem 1997; 272: 1534-40.

[42] Kim MS, Fujiki R, Kitagawa H, et al. 1alpha,25(OH)2D3-induced DNA methylation suppresses the human CYP27B1 gene. Mol Cell Endocrinol 2007; 265-266: 168-73.

[43] Pogribny IP, Miller BJ, James SJ. Alterations in hepatic p53 gene methylation patterns during tumor progression with folate/methyl deficiency in the rat. Cancer Lett 1997; 115: 31-8.

[44] Mass MJ, Wang L. Arsenic alters cytosine methylation patterns of the promoter of the tumor suppressor gene p53 in human lung cells: a model for a mechanism of carcinogenesis. Mutat Res 1997; 386: 263-77.

[45] Monk M, Boubelik M, Lehnert S. Temporal and regional changes in DNA methylation in the embryonic, extraembryonic and germ cell lineages during mouse embryo development. Development 1987; 99: 371-82

[46] Ng RK, Gurdon JB. Epigenetic inheritance of cell differentiation status. Cell Cycle 2008; 7: 1173-7.

[47] Leone G, D'Alò F, Zardo G, et al. Targeted therapy in leukemia. Epigenetic treatment of myelodysplastic syndromes and acute myeloid leukemias. Curr Med Chem 2008; 15: 1274-87.

[48] Maron BA, Loscalzo J. Should hyperhomocysteinemia be treated in patients with atherosclerotic disease? Curr Atheroscler Rep 2007; 9: 375-83. 